\section{BMJ Paediatrics Open}

\title{
Behavioural activation for overweight and obese adolescents with low mood delivered in a community setting: feasibility study
}

\author{
Bronia Arnott, ${ }^{1}$ Charlotte Emma Wray Kitchen (D , ${ }^{2}$ David Ekers, ${ }^{2,3}$ Lina Gega, ${ }^{2}$ \\ Paul Alexander Tiffin ${ }^{4,5}$
}

\begin{abstract}
To cite: Arnott B, Kitchen CEW, Ekers D, et al. Behavioural activation for overweight and obese adolescents with low mood delivered in a community setting: feasibility study. BMJ Paediatrics Open 2020;4:e000624. doi:10.1136/ bmjpo-2019-000624

- Additional material is published online only. To view, please visit the journal online (http://dx.doi.org/10.1136/ bmjpo-2019-000624).
\end{abstract}

Received 13 December 2019 Revised 2 April 2020 Accepted 3 April 2020
Check for updates

(c) Author(s) (or their employer(s)) 2020. Re-use permitted under CC BY-NC. No commercial re-use. See rights and permissions. Published by BMJ.

${ }^{1}$ Population Health Sciences Institute Newcastle University, Newcastle upon Tyne, UK ${ }^{2}$ Department of Health Sciences, University of York, York, UK ${ }^{3}$ Tees Esk and Wear Valleys NHS Foundation Trust, Durham, UK ${ }^{4}$ Department of Health Sciences \& the Hull York Medical School, University of York, York, UK ${ }^{5}$ Tees Esk and Wear Valleys NHS Foundation Trust, Teesside, UK

Correspondence to Dr Bronia Arnott; bronia.arnott@ newcastle.ac.uk

\section{ABSTRACT}

Background Mood and weight problems are common in young people, yet few treatments address both conditions concurrently. Behavioural activation (BA) has shown promise as a treatment for adults with comorbid obesity and depression. This study aimed to examine the feasibility and acceptability of a manualised BA treatment targeting weight and mood problems in young people.

Methods Young people with low mood and weight difficulties were identified via a school-based screening process. Following a diagnostic interview, young people with clinically significant mood problems and concurrent overweight/obesity were invited to participate. A total of 8-12 sessions of BA were delivered by a graduate therapist to eight adolescents (four male) aged 12-15 years. Weight, mood and functioning were assessed before, during and after treatment, and a semistructured qualitative interview was conducted, along with selected outcome measures at 4 months' follow-up.

Results Low attrition and positive qualitative feedback suggested the intervention was acceptable. Trends towards a reduction in reported depression symptoms and improved functioning scores were observed at follow-up, with more mixed results for change in body mass index. Of those attending the 4-month follow-up, 57\% (4/7) no longer met the screening threshold for major depressive disorder. However, low screening and baseline recruitment rates would pose challenges to executing a larger trial. Conclusions BA delivered by a graduate therapist in a British community setting is an acceptable, feasible treatment for comorbid mood and weight problems in adolescence, and its effectiveness should be evaluated in an adequately powered randomised controlled trial.

\section{INTRODUCTION}

In the UK, around $18 \%$ of adolescents aged 11-14years score above the threshold for emotional problems; gender, living in an area of socioeconomic deprivation, status as a child in need and age all increase young people's likelihood of experiencing mental health difficulties, ${ }^{1}$ which are associated with educational underachievement, longterm morbidity, disability and economic costs. ${ }^{23}$ National obesity levels in UK-based
What is known about the subject?

Behavioural activation (BA) has been successfully used with adults with comorbid mood and weight problems.

- Staff with no previous psychotherapy training have delivered BA treatment effectively.

- Young people with depression have found a BA approach useful for improving their mood symptoms.

\section{What this study adds?}

This study explores the feasibility of a BA-based intervention for overweight and obese adolescents with low mood/depression in a UK community setting delivered by a graduate therapist.

- This study found good levels of engagement and acceptability of the intervention in this context.

young people are also high. ${ }^{4}$ Emotional and weight problems may co-occur relatively frequently, with obese young people more than twice as likely to be above the screening threshold for an emotional disorder compared with those who were non-obese. ${ }^{5}$ In adults, concerns have been raised that this dual comorbidity can lead to less weight loss than non-depressed patients during weightloss programmes ${ }^{6}$ perhaps due in part to the shared "maladaptive mood regulatory habits involving eating and inactivity. ${ }^{7}$ This indicates that those with comorbid conditions may benefit from more specialised, tailored interventions.

Adolescence presents the ideal timeframe in which to address detrimental behaviours before they become ingrained; as weight problems are known to persist into adulthood ${ }^{8}$ However, there is limited guidance for treating concurrent mood and weight problems, and existing treatments for addressing obesity and depression individually in young 
people have a number of limitations. The standard first-line psychotherapy for depression is cognitivebehavioural therapy, which may have a more modest impact on depression in young people compared with adults. ${ }^{9}$ Moreover, traditional approaches to managing obesity do not address the underlying links between mood and eating. ${ }^{11}$ One therapy purported to address the common features between obesity and depression is behavioural activation (BA). In BA, individuals are encouraged to focus on the links between mood, eating and behaviour and are supported to spend more time engaging in rewarding activities. One way this could facilitate weight loss may be by helping to increase the frequency of healthy mood regulatory behaviours, breaking the over-reliance on eating as a way of coping with negative moods. ${ }^{12}$ It may be possible to address overeating directly by substituting unhealthy foodrelated behaviour with other rewarding behaviours and also by increasing the time spent in exercise. We anticipate these mechanisms would work in a similar way for adolescents and theorise that treating both conditions in tandem may be more economical. Additionally, BA is well suited to delivery in community settings as it can be delivered by a non-mental health specialist staff. ${ }^{13}$ McCauley and colleagues ${ }^{14}$ examined issues relating to the implementation feasibility and short-term outcomes of a 14-session BA programme in 60 clinically referred adolescents. In this US-based trial, participants undergoing BA showed statistically significant improvements in depression, global functioning and measures of activation and avoidance from pretreatment to end of treatment. However, weight was not assessed as part of this study.

In the USA, a pilot study of BA in working-age adults with comorbid obesity and depression reported significantly reduced weight and self/clinician-rated depression scores, with over $70 \%$ of participants achieving full remission from depression after 12 weeks. ${ }^{12}$ A randomised controlled trial of the same intervention in obese women with major depressive disorder (MDD) found that when BA was delivered prior to a lifestyle intervention, BA improved outcomes in relation to depression but not weight loss at 12 months. ${ }^{6}$ However, the authors did observe an association between improved depression and greater weight loss.

In the UK, early intervention services are often delivered in school or community settings to reduce stigma and cost, and to improve access and reach. ${ }^{15}$ We investigate the acceptability and feasibility of a BA-based intervention for young people with low mood/depression who are also overweight/obese in a UK community sample. We aimed to explore if the BA focus on depression, which is well supported by evidence in adults, was acceptable in young people and offered any opportunity to introduce positive reinforcement of healthy eating behaviours and exercise.

\section{METHODS}

\section{Risk management}

Risk management procedures from the local mental health trust were followed.

\section{Design}

Mixed-methods feasibility study. Participants were assessed using quantitative mood, functioning and weight measures at baseline, 4th, 8th (if relevant) and final sessions. A qualitative interview was undertaken at 4-month follow-up alongside measures of mood and weight.

\section{Setting}

A single, socioeconomically and ethnically diverse secondary school in the North East of England between November 2012 and March 2013.

\section{Procedure}

Patient and public involvement (PPI)

A focus group was held with young people in the local mental health trust to discuss the study design. One example of changes made in light of this input was the addition of vouchers to reimburse participants for their time. The study materials were trialled with young people and their parents/carers and amended in light of their feedback from a small study prior to the start. A PPI youth representative was recruited following this study to advise on dissemination.

\section{Screening}

Information and 'opt-out' forms were sent home in advance to parents/carers. Young people whose parents/ carers returned an opt-out form were not approached to participate in the screening. All remaining young people (in years 8-10) were asked to provide written informed consent to take part in the screening procedure by a researcher. The Patient Health Questionnaire-2 (PHQ-2) screening tool, ${ }^{16}{ }^{17}$ with an additional help-seeking question, was completed in registration class by those present on the day of screening (years 8-10). Body Mass Index (BMI) was self-reported or assessed by a member of the research team on the day in a private room. To reflect the younger age of those in year 7 , the information and screening materials were sent to families so they could be completed by young people at home with parents/carers.

The BMI was calculated as kilogram per square metre adjusted for age and sex; an extended International Obesity Task Force (IOTF) BMI classification was used where ' 1 ' is 'overweight' and ' 2 ' is 'very overweight/ obese'. Those with overweight, as well as obesity, were included to reflect the early intervention model. A BMI z-score, a measure of relative weight adjusted for child age and sex, was also reported. The PHQ-2 inquires about the frequency of depressed mood and anhedonia over the past 2 weeks; scores range from 0 to $6 .{ }^{16}$ A score of 3 has been identified as the optimal cut-off to screen for depression. ${ }^{17}$ 
Students with obesity/overweight and comorbid low mood (PHQ-2>3) who were interested in receiving help were invited by letter to an individual baseline assessment.

\section{Baseline assessments and measures}

Young people, their parents/carers, a graduate therapist and consultant adolescent psychiatrist attended the diagnostic assessment, which was completed in a private room in a community hall on the school site. Young people were offered a $£ 10$ voucher for attending this session. Prior to the start of the diagnostic interview, young people over 16years old provided written informed consent and young, people under 16 years old provided written informed assent (and their parents/carers provided written informed consent).

The Kiddie-Sads-Present and Lifetime Version $\left(\mathrm{K}-S A D S-\mathrm{PL}^{18}\right)$, a validated clinical structured interview aligned to the Diagnostic and Statistical Manual of Mental Disorders, 5th Edition, criteria, was administered to young people (and subsequently their parents/carers if under 16) by a consultant adolescent psychiatrist (and doubled coded by a graduate therapist). Clinical judgement was used to resolve discrepancies. The K-SADS-PL is a semistructured interview aimed at diagnosing current and past clinical disorders, one of which is MDD and associated subcategories; it takes between 45 and 75 min to administer. ${ }^{18}$ We focused only on current diagnoses. Additional measures of low mood (Mood and Feelings Questionnaire-Short Form (MFQ-SF $\left.{ }^{19}\right)$ ), weight (BMI), general health and social functioning (Health of the Nation Outcome Scales for Children and Adolescents $\left(\mathrm{HoNOSCA}^{20}\right)$ ) and functioning (Children's Global Assessment Scale $\left(\mathrm{CGAS}^{18}\right)$ ) were administered. The MFQ-SF is a 13-item questionnaire based on Diagnostic and Statistical Manual of Mental Disorders, Third Edition, Revised, criteria for depression, consisting of a series of descriptive phrases regarding how the young person has been feeling/acting over the last 2 weeks. ${ }^{19}$ The 15 -item HoNOSCA focuses on general health and social functioning of young people (rating on a scale of $0-4$ ) and is clinician rated. ${ }^{20}$ The CGAS is administered as part of the K-SADS-PL and involves a number (between 0 and 100) being allocated by the clinician using descriptor categories to describe young people's level of functioning. ${ }^{18}$

Young people were eligible (and entered the study) if they met the diagnostic criteria for MDD or depressive disorder not otherwise specified (DD-NOS) on the K-SADS-PL or were deemed to have significant depressive symptoms by the consultant adolescent psychiatrist. The purpose of the diagnostic interview was to provide a 'gold standard' diagnosis for all intervention study participants and to assess the feasibility of this approach (and appropriate thresholds for a community setting) to inform the design of a future trial. Those without a clinical depression diagnosis were included for this purpose. Participants were excluded under the following circumstances: (1) currently undergoing psychotherapy, (2) presence of psychotic/manic symptoms, (3) significant active substance abuse/dependence, and (4) presence of current or history of serious self-harm or acute suicidality. The exclusion criteria reflected the community setting and relative inexperience of the practitioner. Feasibility was assessed by considering screening, baseline assessment and intervention recruitment rates, as well as participant, parental/carer and therapist satisfaction. Attendance was recorded at each session and a qualitative interview explored satisfaction and acceptability following treatment.

Eligible individuals were then randomised by an independent member of the research team using a computerised permuted block method (block size of 4) to the BA intervention or waiting list control (4-6weeks wait prior to the BA intervention). The purpose of randomisation was to explore the acceptability of being allocated to a waiting list control and to spread the caseload of the graduate therapist across the academic year. All participants received the BA intervention and data were not analysed by group. As such, allocation concealment was not essential in this study.

\section{Manual and intervention development}

The participant treatment manual (named the 'body and mind manual') for young people and their parents / carers and an associated guide for practitioners were adapted from material tested informally in a small sample of young people in North America ${ }^{21}$; a subsequent version of this American manual was used in the aforementioned trial of the intervention. ${ }^{14}$ Further content for the body and mind participant treatment manual and practitioner guide, in particular, were sourced from manuals used in a trial of BA in depressed adults. ${ }^{22}$ The consultant adolescent psychiatrist and graduate therapist developed the body and mind manual to be suitable for young people in the UK, with input from an expert in $\mathrm{BA}$ and a developmental psychology researcher trained in BA. Our intervention focused on early intervention for young people with mild to moderate mood difficulties (both those with and those 'at risk' of depression) while also being overweight or obese. The body and mind materials and training were adapted to be delivered by a graduate therapist with no previous psychotherapy experience, in a school setting, supervised by an experienced consultant adolescent psychiatrist. The adapted materials in the body and mind manual were used in a previous BA study by members of the research team and were subsequently adapted in light of PPI feedback from clinicians, young people and parents/carers. The body and mind manual provided therapist intervention session guidance and worksheets for young people (and their parents/ carers if present) to complete in and between sessions. The topics for each session have been included in online supplementary appendix 1 , and further information can be obtained from the corresponding author. No modifications were made during the study to the manual, but following the qualitative feedback, adaptions were made to the manual materials. 


\section{Intervention delivery}

A maximum of 12 weekly, 1 hour, manualised BA sessions were delivered face-to-face via individual appointments after school hours by a graduate therapist who had attended a 5-day BA training course. Treatment was delivered using paper materials. The number of sessions offered was at the discretion of the therapist (minimum of 8), and the order/content could be tailored to individuals. Parents/carers were encouraged to participate in sessions with adolescent agreement. Each session started with an agenda-setting task and included activities for young people, their parents/carers either together or separately. Each treatment was individualised based on the findings of the previous session(s) and generally consisted of task setting or worksheet completion. The intervention was designed to work to address barriers to improving mood/weight difficulties and developing personalised strategies to support these aims following a BA model. Vouchers $(£ 10)$ were given to participants at fourth, eighth and final sessions.

Weekly supervision sessions were held during the delivery of the intervention between the graduate therapist and consultant adolescent psychiatrist; in addition to this, the psychiatrist provided risk cover throughout the study.

\section{Fidelity}

A random 10\% of treatment sessions were audio-recorded and coded for fidelity by a BA specialist who was blind to information about the participants. Fidelity materials were developed in a previous BA trial for adults. ${ }^{22}$

\section{Follow-up}

Quantitative measures (CGAS, MFQ-SF, HoNOSCA and BMI) were administered at baseline, at 4 and 8 weeks (if relevant), and final session (end of treatment). Measures of weight (BMI) and mood (MFQ-SF) were re-administered at the 4-month (16week) follow-up, along with a qualitative interview.

\section{Qualitative interview}

Information was captured in brief semistructured interviews with young people, their caregivers and the graduate therapist. Interviews followed a topic guide (see online supplementary appendix 2) which covered screening, baseline assessment, intervention, follow-up, and future research and treatment. Interview notes were coded into themes, categories, subcategories and $\operatorname{codes}^{23}$ using an inductive framework approach.

\section{Data analysis}

We examined and reported recruitment rates and outcome measures using percentages and summary statistics. Results were not reported or analysed in the groups that they were assigned during randomisation; rather, for those allocated to treatment immediately, their diagnostic assessment was treated as their baseline data, and for those allocated to the waiting list, an assessment taken

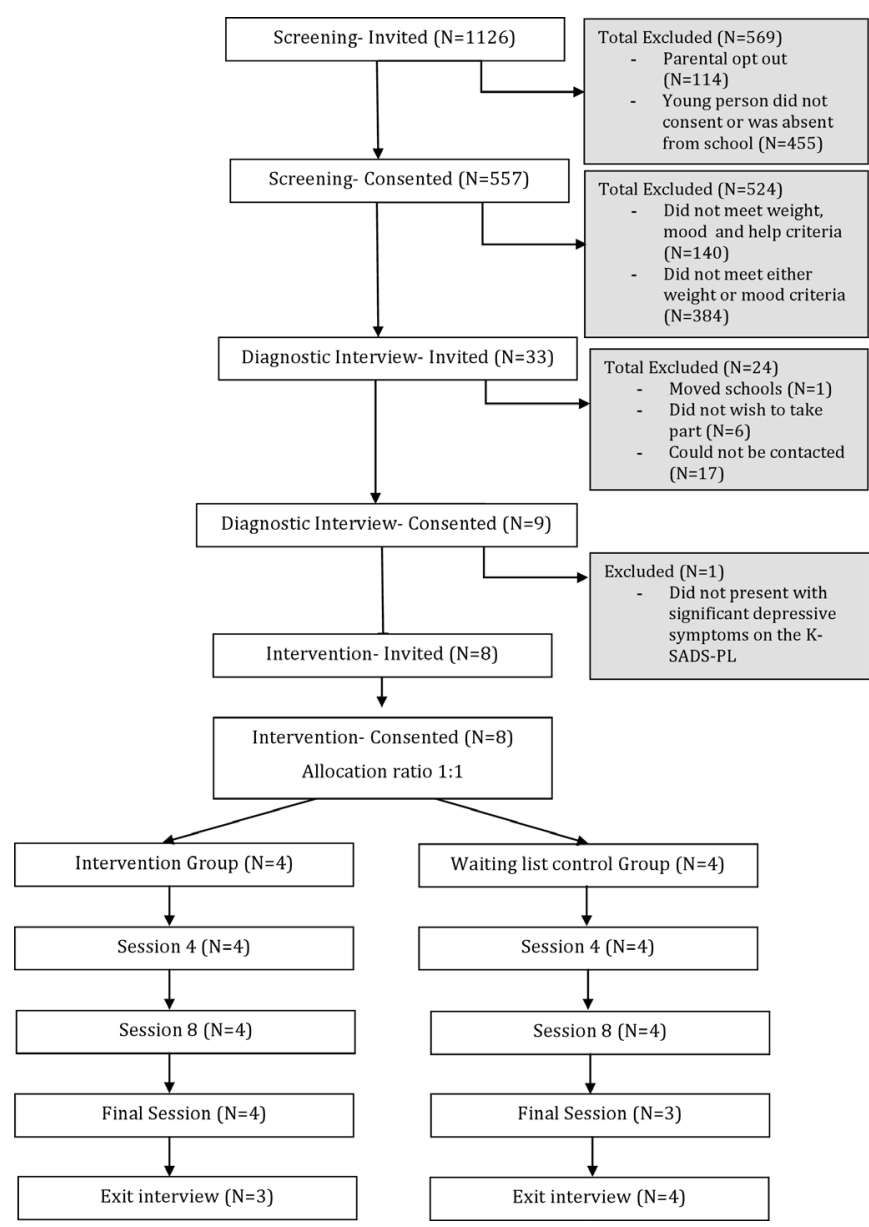

Figure 1 Consolidated Standards of Reporting Trials diagram of participant recruitment and retention. K-SADSPL, Kiddie-Sads-Present and Lifetime Version.

prior to their first session was used (rather than the measures taken at study entry).

\section{RESULTS}

\section{Recruitment}

The school approached to participate agreed; figure 1 shows participant identification and recruitment; a total of 1126 pupils were invited to the initial screening with 557 participating (49\%) over a period of 4 weeks; 503 $(90 \%)$ of these were recruited via in-person screening and $54(10 \%)$ from postal screening. Those who did not participate were either absent or did not wish to participate $(n=455)$ or were withdrawn by their parents ( $n=114$ ). After screening, 33 (representing $3 \%$ of those invited to take part or $6 \%$ of those who participated in the screening) were invited to diagnostic interview; 9 attended (27\% of those provisionally eligible). Ineligible responders either were not obese/overweight $(n=122)$, had no mood difficulties $(n=18)$ or had neither weight or mood difficulties $(\mathrm{n}=382)$. Two additional young people had mood and weight difficulties (met the inclusion criteria) but did not want help with these difficulties (indicated by ticking no to the help-seeking question). 
Of the nine, eight were eligible at the diagnostic interview stage (1.4\% of those taking part in the screening procedure); one participant was excluded at this stage as he or she did not have clinically significant mood symptoms and so did not meet the study inclusion criteria. All eight young people identified as eligible agreed to be randomised and to participate in the intervention.

Four participants were randomised to BA immediately and four to a waiting list control. The sample included two sets of siblings. All eight eligible young people (mean age $=14.27$ years, $\mathrm{SD}=0.97$; four male; $7 / 8$ white) participated in the intervention and were followed up between 15 and 32 weeks postintervention. Delays in follow-up appointments (from the intended 16weeks) reflected the availability of participants and researcher.

\section{Intervention}

Participating young people completed between 8 and 11 sessions of $\mathrm{BA}$, with seven (out of eight) adolescents completing all BA sessions prescribed by the therapist. All coded treatment sessions were judged to be adhering to the BA model and manual. Session attendance was high; one participant did not attend the final session but attended the follow-up session, and a further participant was unavailable for the follow-up assessment. Some sessions were rearranged or delivered in tandem due to illness, missed appointments or transport difficulties. No significant events, harm or unintended effects were recorded. Participant quantitative outcome measures are shown in table 1.

\section{Low mood and functioning}

Atbaseline, according to the K-SADS-PL, seven participants reached the criteria for a clinically significant depressive disorder (6 MDD and 1 DD-NOS). The remaining participant had significant low mood symptoms not reaching the criteria for a diagnosis of a clinical mood disorder. At baseline, the mean MFQ-SF score was 10.38 (SD=6.67) above the screening threshold for MDD $(\geq 8)$; the mean CGAS score was 66.13 ( $\mathrm{SD}=6.2$ ), indicating 'some problems' in one area only; and the mean HoNOSCA score was $10.38(\mathrm{SD}=4.50)$, indicating a number of problems in various areas. This study was not intended to be powered to detect a clinically or statistically significant treatment effect. Therefore, trends in relation to the outcomes of interest are reported for descriptive purposes, though not formally tested for intergroup differences. In this respect, self-report MFQ-SF scores improved in all but one participant from baseline to end of treatment, where their score remained the same, reflecting an effect size (estimated by the difference in total MFQ-SF score) of 0.58 . At the 4-month follow-up, the results were more mixed, but the effect size was 0.16 . An MFQ-SF cut-off score of 7 or below indicated remission, which was achieved in five cases at the end of treatment (one young person did not attend the final session). In the remaining two cases, a decrease in MFQ-SF score was evident. At follow-up, remission was maintained in all but one of those who had achieved it at the end of treatment (one was unable to attend follow-up). In the remaining cases, one young person had subsequently achieved remission and two had not. Therapist-reported CGAS ratings improved in all cases from baseline to end of treatment (one young person did not attend the final session). An effect size of 1.26 was observed for the improvements in C-GAS scores at end of treatment. Summed HoNOSCA scores generally improved with an effect size of 1.22 at the end of treatment.

\section{Weight}

The average raw $\mathrm{BMI}$ at baseline was $28.98(\mathrm{SD}=4.98)$ and the mean BMI z-score (relative weight adjusted for child age and sex) was $2.42(\mathrm{SD}=0.66)$, equating to four young people who were obese and four who were overweight according to IOTF classifications. BMI decreased in two participants and increased in five participants from baseline to end of treatment (one did not attend follow-up). Participant BMI had decreased between the end of treatment and the 4-month follow-up for four but not for two (two did not attend follow-up/final session). Preliminary analyses suggested that, overall, from baseline to end of treatment, there was an effect size of 0.19 recorded for raw BMI and an effect size of 0.18 for BMI z-score.

\section{Acceptability}

Seven qualitative interviews with young people (alongside parents/carers in two cases) and the graduate therapist were conducted, lasting between 20 and $30 \mathrm{~min}$. An overview of the qualitative themes emerging from the interviews is provided in table 2.

Young people reported high levels of satisfaction with content, setting, frequency and duration of the sessions. Participating adolescents valued confidential discussions with the therapist and goal-related tasks. Some young people reported significant and sustained improvements in mood and self-esteem. Those who reported more difficulty in maintaining gains perceived the challenges as mainly related to external barriers. Young people suggested that they may benefit from top-up BA sessions to help them sustain changes.

When asked about future study design, young people expressed a willingness to undergo randomisation to individual sessions of BA, or other psychological therapies, or indeed to a waiting list control. Participants were less keen to be randomised to a medication treatment arm or to a group-based intervention. Adolescents viewed the screening process positively, though concerns were expressed about being weighed and asked about mood in a school setting.

Participating parents reported that they had found the intervention highly acceptable. Again, goal-oriented activities were perceived as particularly beneficial. The non-specialist therapist was perceived as highly acceptable. Parental difficulty in participating due to other commitments was highlighted as a feasibility issue. They perceived a positive effect of the intervention on their 


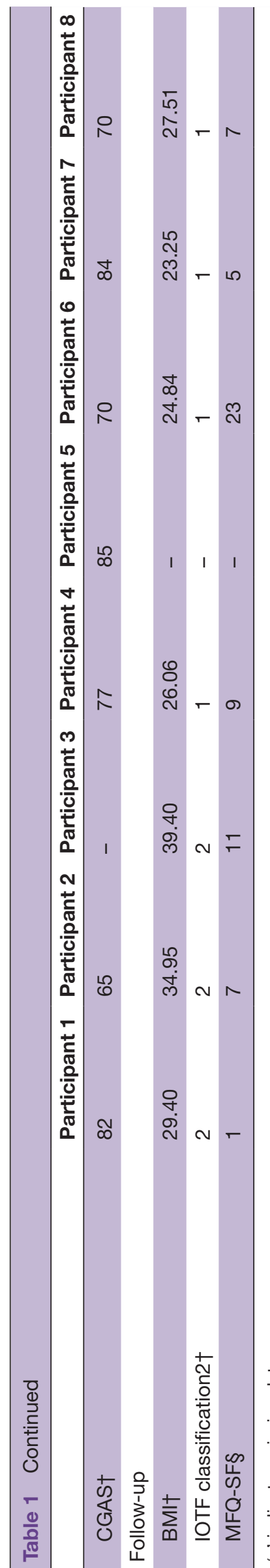

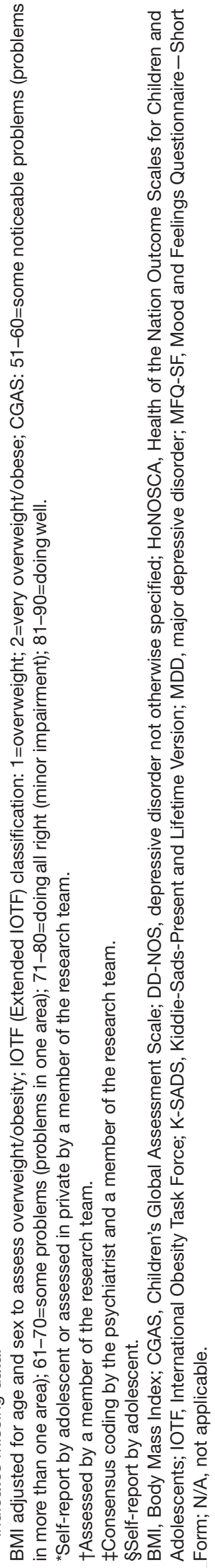

child's mood and self-esteem. Parents also reported a positive effect of the intervention on their own selfesteem and reported their adolescents had changed their food-related behaviours, although often the wider family had not. The graduate therapist noted difficulties with session scheduling due to school holidays.

\section{DISCUSSION}

We explored the feasibility of a BA intervention as a treatment for comorbid low mood and overweight/ obesity in young people in England. As in previous work in adolescents with low mood, BA was found to be feasible to implement ${ }^{14}$ and acceptable to young people and parents/carers ${ }^{24}$ evidenced by the low attrition rates and qualitative findings. In line with previous research, this study provided tentative suggestions that the intervention may improve mood and functioning. This study extends the BA literature by applying this promising treatment to an adolescent population in a community setting with comorbid mood and weight difficulties. Our results support the feasibility and acceptability of BA in this context. This research is timely, as evidenced by the recent National Institute for Health and Care Excellence research recommendations highlighting BA as a key area for future research, alongside consideration of non-clinical settings for intervention delivery. ${ }^{25}$ Evidence for the treatment of depression using BA prior to treatment for comordid obesity in clinical settings with adults is mounting, ${ }^{6} 12$ emphasising the potential benefits of tackling mood prior to weight issues. However, our study suggested more mixed results for weight.

Although we showed that it is possible to recruit and randomise young people to BA or a waiting list control, recruiting in a school setting was a challenge. The resource intensive nature of school-wide screening was not feasible due to the initial low uptake $(49 \%)$ from the pool of invited participants and also the final yield of provisionally eligible participants $(6 \%)$, resulting in a high investment of time and resources for a limited return, in terms of final numbers of participants. Paper materials and researcher time for completing this screening process were also costly. These findings were in line with evidence from a systematic review of the feasibility of school-based identification of young people experiencing or at-risk of developing mental health difficulties, which also found cost, time, resources and obtaining consent were common barriers. ${ }^{26}$ It is important to note that in-person screening was more successful than postal screening; this may reflect the younger age of those contacted via postal screening, although the stark difference suggests this is not the case. Interestingly, our findings did support previous research $^{5}$ that suggested it was more common to have mood difficulties without weight problems than it was to have weight problems without low mood. This may be an important consideration when targeting such young people for future similar interventions. It may 
Table 2 Qualitative themes and example quotes from interviews

Theme Example quote

\begin{tabular}{|c|c|c|}
\hline \multicolumn{3}{|l|}{ Screening process } \\
\hline & Pressure & '(school) wanted us to do it' \\
\hline & Choice & '(wouldn't get weighed) in front of mates' \\
\hline & Stigma & '(getting weighed felt) awkward’' \\
\hline \multicolumn{3}{|l|}{ Baseline } \\
\hline & 'Being different' and sensitive handling & '(being invited back was) nerve wracking' \\
\hline & Information and personal choice & $\begin{array}{l}\text { 'knew I might have to wait a while (for treatment if } \\
\text { in waiting list control group)' }\end{array}$ \\
\hline \multicolumn{3}{|l|}{ Intervention } \\
\hline & $\begin{array}{l}\text { Personal investment and internal versus } \\
\text { external factors }\end{array}$ & $\begin{array}{l}\text { 'talked about what I wanted to talk about (during } \\
\text { sessions)' }\end{array}$ \\
\hline & Individual choice & 'didn’t want mum to come (to sessions)' \\
\hline \multicolumn{3}{|c|}{ Future research/treatment } \\
\hline & Responsibility to help & $\begin{array}{l}\text { 'would like to do it (future studies) to make sure it } \\
\text { could help others' }\end{array}$ \\
\hline & Personal choice & 'prefer this kind of thing (to medication)' \\
\hline
\end{tabular}

be possible that future trials and implementation could take advantage of existing opt-out health screening initiatives which are already embedded in schools (eg, National Child Measurement Programme in year 6) to screen large numbers of children for overweight and low mood. Discretion for the young person will be key due to the stigma surrounding overweight and low mood, as highlighted in the qualitative interviews.

Although modest numbers of young people $(n=33)$ were identified as provisionally eligible for the study, very few of those invited to the diagnostic interview attended $(n=9)$. One limitation of this study is that the qualitative investigation did not seek to investigate why this might be the case. One possibility is that the stigma participating young people alluded to surrounding the screening process may have been a contributing factor. Alternatively, the study materials or recruitment approach may not have been favourable. Despite this, the structured diagnostic interview and associated outcome measures appeared acceptable and did not lead to dropout. However, no measures of diet or physical activity outcomes were collected; BMI was the only weight metric, which may not have been sensitive enough to demonstrate change over the relatively short timescale of the study. Future studies should use a longer follow-up and regular measures of diet/activity in order to better assess the impact of the intervention. Although follow-up appointments were intended to be scheduled 16 weeks following study entry, in reality, they took place between 15 and 32 weeks due to participant/researcher availability. This would need to be addressed in a trial.

Once participants were recruited to the intervention phase, attendance at BA sessions was high; the feedback on the intervention from young people, the therapist and parents/carers was encouraging; and the outcomes were generally positive. One suggestion for improvement was the staggering of sessions towards the end of treatment or the addition of 'top-up' BA sessions following the end of treatment; this may help bridge the gap between reliance on the therapist and independence at home.

This study had a number of limitations. We included only a small sample of young people in a single school, and while there was some variation in terms of gender and ethnicity, the representativeness of the results is limited. This should be considered when planning future trials. A single therapist delivered the intervention, so it was not possible to generalise to other practitioners. The participants were drawn from a community population and therefore may have been less severely depressed and had fewer attendant risks than young people seen in secondary or tertiary services. Although it was not the intention of this study to assess the ability of the nonspecialist therapist to deal with patients who are more at risk, a trial in depressed working-age adults does suggest that BA can be delivered by non-specialists to even quite unwell patients in a way which is both clinically and costeffective. ${ }^{27}$ Participants were offered vouchers to participate following PPI advice; this would be costly in a trial and may represent a source of bias. However, future researchers may wish to consider this option, as it was supported by our ethics committee and it is common practice to enhance retention rates in large clinical trials. Finally, the data in the study are several years old; however, it is important that these data are published to avoid duplication of research efforts and to inform the direction of future research, particularly in light of recent policy changes surrounding mental health provision in schools. ${ }^{28} 29$ 


\section{CONCLUSIONS}

Comorbid depression and obesity present a concerning public health issue. Findings from this study suggest that BA delivered in a community setting by a nonspecialist may be a promising, non-stigmatising intervention for young people affected by comorbid depressive symptoms and overweight. In the UK, there is increasing pressure to prioritise well-being within schools $^{28} 29$ to address mental and physical health in children and adolescents. Our findings suggest that a manualised BA-based intervention may represent an effective approach to mood and weight problems in young people that could be delivered by non-specialist practitioners. Such an approach should be evaluated via an adequately powered randomised controlled trial, addressing effectiveness and cost-effectiveness. If young people were screened in national initiatives (eg, NCMP) for overweight and low mood and then referred into a community BA intervention programme delivered by a non-specialist, this would hopefully increase detection, decrease treatment waiting times and reduce stigma, and also be more cost-effective and replicable than the school-based screening method employed in this study.

Acknowledgements We thank the young people, parents/carers and school staff for their help with the study. We are indebted to Elizabeth McCauley and colleagues at the University of Washington, Seattle and the COBRA team at Tees, Esk and Wear Valleys NHS Trust for sharing their Behavioural Activation manuals for adaptation.

Contributors BA, DE and PAT were responsible for study design. DE was responsible for delivery of the behavioural activation training and fidelity. CEWK delivered the intervention under supervision from PAT and DE. BA and CEWK were both responsible for data collection. All authors were responsible for data analysis and the drafting of the manuscript.

Funding This study was funded by an award from the Health Education Innovation Cluster North-East (UK) to the first and last authors with matched funding from Durham University. The funder played no role in the design of the study or collection, analysis or interpretation of data. PAT is supported in his research by a National Institute for Health Research (NIHR) Career Development Fellowship. The views expressed are those of the authors and not necessarily those of the NHS, the NIHR or the Department of Health.

Competing interests No, there are no competing interests.

Patient consent for publication Not required.

Ethics approval Approvals were obtained from Durham University Ethics Advisory Sub-Committee, Department of Psychology (reference 10/26). NHS ethical approvals were not required for this study, which took place in a community setting.

Provenance and peer review Not commissioned; externally peer reviewed.

Data availability statement All data relevant to the study are included in the article or uploaded as supplementary information. All relevant data is included in the manuscript and supplementary material. Paper consent forms have been securely archived by Durham University.

Open access This is an open access article distributed in accordance with the Creative Commons Attribution Non Commercial (CC BY-NC 4.0) license, which permits others to distribute, remix, adapt, build upon this work noncommercially, and license their derivative works on different terms, provided the original work is properly cited, appropriate credit is given, any changes made indicated, and the use is non-commercial. See: http://creativecommons.org/ licenses/by-nc/4.0/.

ORCID iD

Charlotte Emma Wray Kitchen http://orcid.org/0000-0002-9323-0061

\section{REFERENCES}

1 Deighton J, Lereya ST, Casey P, et al. Prevalence of mental health problems in schools: poverty and other risk factors among 28000 adolescents in England. Br J Psychiatry 2019;215:565-7.

2 Dunn V, Goodyer IM. Longitudinal investigation into childhoodand adolescence-onset depression: psychiatric outcome in early adulthood. Br J Psychiatry 2006;188:216-22.

3 Green $\mathrm{H}$, McGinnity A, Meltzer H, et al. Mental health of children and young people in Great Britain, 2004.

4 Stamatakis E, Wardle J, Cole TJ. Childhood obesity and overweight prevalence trends in England: evidence for growing socioeconomic disparities. Int J Obes 2010;34:41-7.

5 Tiffin PA, Arnott B, Moore HJ, et al. Modelling the relationship between obesity and mental health in children and adolescents: findings from the health survey for England 2007. Child Adolesc Psychiatry Ment Health 2011;5:31.

6 Pagoto S, Schneider KL, Whited MC, et al. Randomized controlled trial of behavioral treatment for comorbid obesity and depression in women: the be active trial. Int J Obes 2013;37:1427-34.

7 Pagoto SL, Spring B, Cook JW, et al. High BMI and reduced engagement and enjoyment of pleasant events. Pers Individ Dif 2006;40:1421-31.

8 Simmonds M, Llewellyn A, Owen CG, et al. Predicting adult obesity from childhood obesity: a systematic review and meta-analysis. Obes Rev 2016;17:95-107.

9 Dubicka B, Elvins R, Roberts C, et al. Combined treatment with cognitive-behavioural therapy in adolescent depression: metaanalysis. Br J Psychiatry 2010;197:433-40.

10 Weisz JR, McCarty CA, Valeri SM. Effects of psychotherapy for depression in children and adolescents: a meta-analysis. Psychol Bull 2006;132:132-49.

11 Ebbeling CB, Pawlak DB, Ludwig DS. Childhood obesity: publichealth crisis, common sense cure. Lancet 2002;360:473-82.

12 Pagoto S, Bodenlos JS, Schneider KL, et al. Initial investigation of behavioral activation therapy for co-morbid major depressive disorder and obesity. Psychotherapy

13 Ekers D, Webster L, Van Straten A, et al. Behavioural activation for depression; an update of meta-analysis of effectiveness and sub group analysis. PLoS One 2014;9:e100100.

14 McCauley E, Gudmundsen G, Schloredt K, et al. The adolescent behavioral activation program: adapting behavioral activation as a treatment for depression in adolescence. J Clin Child Adolesc Psychol 2016;45:291-304.

15 McGorry PD, Mei C. Early intervention in youth mental health: progress and future directions. Evid Based Ment Health 2018;21:182-4.

16 Whooley MA, Avins AL, Miranda J, et al. Case-Finding instruments for depression. two questions are as good as many. J Gen Intern Med 1997:12:439-45.

17 Manea L, Gilbody S, Hewitt C, et al. Identifying depression with the PHQ-2: a diagnostic meta-analysis. J Affect Disord 2016;203:382-95.

18 Kaufman J, Birmaher B, Brent D, et al. Schedule for affective disorders and schizophrenia for school-age Children-Present and lifetime version (K-SADS-PL): initial reliability and validity data. J Am Acad Child Adolesc Psychiatry 1997;36:980-8.

19 Angold A, Costello EJ, Messer SC, et al. The development of a short questionnaire for use in epidemiological studies of depression in children and adolescents. Int $J$ of Meth Psych Res 1995;5:237-49.

20 Gowers SG, Harrington RC, Whitton A, et al. Brief scale for measuring the outcomes of emotional and behavioural disorders in children. health of the nation outcome scales for children and adolescents (HoNOSCA). Br J Psychiatry 1999;174:413-6.

21 McCauley E. Behavioural activation manual, 2011.

22 Ekers D, Richards D, McMillan D, et al. Behavioural activation delivered by the non-specialist: phase II randomised controlled trial. Br J Psychiatry 2011;198:66-72.

23 Graneheim UH, Lundman B. Qualitative content analysis in nursing research: concepts, procedures and measures to achieve trustworthiness. Nurse Educ Today 2004;24:105-12.

24 Jacob M, Keeley ML, Ritschel L, et al. Behavioural activation for the treatment of low-income, African American adolescents with major depressive disorder: a case series. Clin Psychol Psychother 2013;20:87-96.

25 NICE guideline. National Institute for health and care excellence (2019) depression in children and young people: identification and management. Available: https://www.nice.org.uk/guidance/ng134/ chapter/Recommendations-for-research

26 Soneson E, Howarth E, Ford T, et al. Feasibility of school-based identification of children and adolescents experiencing, or at-risk of 
developing, mental health difficulties: a systematic review. Prev Sci 2020. doi:10.1007/s11121-020-01095-6

27 Richards DA, Ekers D, McMillan D, et al. Cost and outcome of behavioural activation versus cognitive behavioural therapy for depression (cobra): a randomised, controlled, non-inferiority trial. Lancet 2016;388:871-80.
28 Minds Y. Wise up: prioritising wellbeing in schools, 2017. Available: https://youngminds.org.uk/resources/policy-reports/wise-upprioritising-wellbeing-in-schools/

29 Department of Health and Social Care and Department for Education. Government Response to the Consultation on Transforming Children and Young People's Mental Health Provision: a Green Paper and Next Steps, 2018. Available: www. gov.uk/government/publications 1 